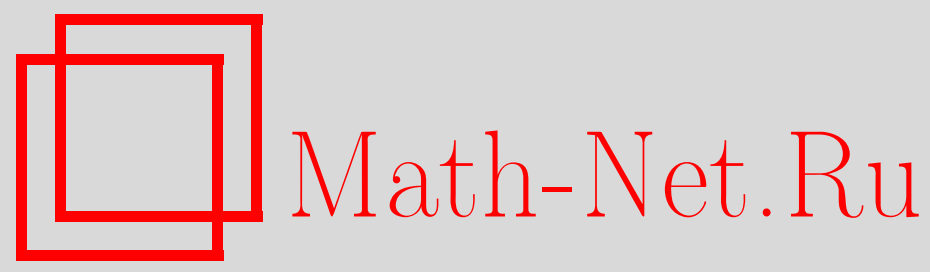

А. В. Угланов, Четыре контрпримера к теореме Фубини, Матем. заметки, 1997, том 62, выпуск 1, 124-127

DOI: https://doi.org/10.4213/mzm1595

Использование Общероссийского математического портала Math-Net.Ru подразумевает, что вы прочитали и согласны с пользовательским соглашением http://www.mathnet.ru/rus/agreement

Параметры загрузки:

IP: 54.237 .59 .107

26 апреля 2023 г., 11:51:07

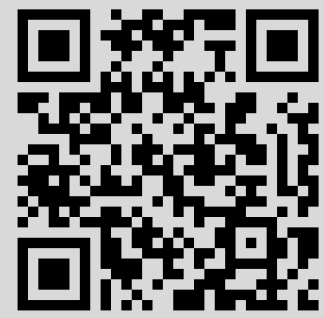


УДК 517

\title{
ЧЕТЫРЕ КОНТРПРИМЕРА К ТЕОРЕМЕ ФУБИНИ
}

\author{
А.В. Угланов
}

Работа относится к теории знаконеопределенных мер. Главные результаты: теорема Фубини в общем виде не верна; жордановы составляющие переходной меры не обязаны быть переходными мерами; операция взятия жордановых составляющих не обязана коммутировать с операцией умножения на начальную меру; произведение $\sigma$-ограниченных мер может не быть $\sigma$-ограниченной мерой.

Библиография: 2 названия.

В работе [1] в весьма общей форме была доказана теорема Фубини; грубо говоря, теорема устанавливает равенство

$$
\int_{X \times Y} f(x, y) \nu \times \mu(d x, d y)=\int_{X}\left[\int_{Y} f(x, y) \nu(x, d y)\right] \mu(d x) .
$$

Здесь $X, Y$-измеримые пространства, $f$-вектор-функция на $X \times Y, \nu$-переходная мера из $X$ в $Y, \mu$-вектор-мера на $X$. (Одна из мер $\nu, \mu$ должна быть скалярной. В настоящей работе вектор-меры и функции затрагиваться не будут, поэтому точных определений и формулировок мы не приводим. Необходимые же скалярные понятия будут даны.) Но если в скалярном знакопостоянном случае $(\nu, \mu$ - скалярные неотрицательные меры, $f-$ скалярная функция) теорема Фубини доказьвается просто, причем в исчерпьвающей общности ( $X, Y$ - произвольные измеримые пространства, $\nu, \mu$ - произвольные меры), то в векторном случае доказательство достаточно сложно и, главное, пришлось делать специальные предположения относительно $X, Y, \nu, \mu$. В [1] отмечалось, что вопрос о справедливости теоремы Фубини в общем случае остается открытьм. Приводимый ниже пример 1 дает отрищательньй ответ на этот вопрос: уже в скалярном, но знакопеременном случае теорема Фубини в общем виде неверна. Примеры 2,3 , фактически также опровергающие этот вид теоремы Фубини, носят более общий характер; они дают отрицательный ответ на интересные и давно стоявшие вопросы: должны ли жордановы составляющие переходной меры быть переходными мерами? Если составляющие все же являются переходными мерами, то должна ли операция взятия составляющих коммутировать с операцией произведения переходной меры на начальную? Несколько особняком стоит пример 4: хотя он и не опровергает теорему Фубини, но показьвает, что пользоваться ей нужно с известной осторожностью: произведение $\sigma$-ограниченных мер может не быть $\sigma$-ограниченной мерой.

Уточним используемые в статье понятия. Пусть $\left(x, \Sigma_{X}\right),\left(Y, \Sigma_{Y}\right)$ - измеримые пространства, $\left(\Sigma_{X}, \Sigma_{Y}-\sigma\right.$-алгебры). Мерой (числовой) на $X$ назьвается счетно-аддитивная функция $\mu: \Sigma_{X} \rightarrow \mathbb{R}^{1}$; положительной мерой на $X$ называется счетно-аддитивная 
функция $\mu: \Sigma_{X} \rightarrow[0, \infty]$ (т.е. $\mu$ может принимать значение $+\infty$ ). Переходной (положительной переходной) мерой из $X$ в $Y$ назьвается функция $\nu: X \times \Sigma_{Y} \rightarrow \mathbb{R}^{1}$ (соответственно, $\left.\nu: X \times \Sigma_{Y} \rightarrow[0, \infty]\right)$, удовлетворяющая условиям:

1) при любом $x \in X$ функция $\nu(x, \cdot)$ есть мера (положительная мера) на $X$;

2) при любом $A \in \Sigma_{Y}$ функция $\nu(\cdot, A)$ измерима.

Множество мер на $X$ обозначим через $M(X)$, множество переходных мер из $X$ в $Y$ обозначим через $M(X, Y)$; такой же смысл для соответствующих положительных объектов имеют обозначения $M^{+}(X), M^{+}(X, Y)$.

Для $\mu \in M(X)$ через $|\mu| \in M(X)$ будем обозначать меру, являющуюся полным изменением меры $\mu$. Все рассматриваемые ниже интегралы - лебеговы; при этом функция $f: X \rightarrow \mathbb{R}^{1}$ называется $\mu$-интегрируемой, если она $|\mu|$-интегрируема, интеграл $\int_{X} f d \mu$ определяется естественным образом как разность интегралов по положительной и отрицательной (жордановьп) частям меры $\mu$.

Для $x \in X, A \subset X \times Y$ положим $S_{x}(A)=\{y \in Y:(x, y) \in A\}$. Предположим, меры $\mu \in M(X)$ и $\nu \in M(X, Y)$ таковы, что при любом $A \in \Sigma_{X} \times \Sigma_{Y}$ функция $x \mapsto \nu\left(x, S_{x}(A)\right)$ (автоматически являющаяся $\Sigma_{X}$-измеримой) $\mu$-интегрируема; тогда положим

$$
\nu \times \mu: \Sigma_{X} \times \Sigma_{Y} \rightarrow \mathbb{R}^{1}: A \mapsto \int_{X} \nu\left(x, S_{x}(A)\right) \mu(d x) .
$$

Известно [1], что функция $\nu \times \mu$ есть мера на $X \times Y$.

Напомним еше, что $\sigma$-алгебра называется сепарабельной, если она порождается некоторым счетным семейством множеств.

Переформулируем теперь применительно к скалярному случаю результат из [1].

Теорема (Фубини). Пусть $\Sigma_{Y}$ - сепарабельная $\sigma$-алгебра, $\mu \in M(X), \nu \in M(X, Y)$, произведение $\nu \times \mu$ определено и функиия $f: X \times Y \rightarrow[-\infty, \infty]$ является $\nu \times \mu$-интегрируемой. Тогда $|\mu|$-почти при всех $x$ функиия $y \mapsto f(x, y) \nu(x, \cdot)$-интегрируема, функиия

$$
x \mapsto \int_{Y} f(x, y) \nu(x, d y)
$$

н-интегрируема и справедливо равенство (1).

При доказательстве теоремы существенно использовались установленные в [1] (в предположениях теоремы) соотношения

$$
\begin{gathered}
|\nu| \in M(X, Y), \\
|\nu \times \mu|=|\nu| \times|\mu| .
\end{gathered}
$$

Среди условий теоремы (и подавно соотношений $(3),(4))$ лишним может быть только одно - условие сепарабельности $\Sigma_{Y}$; мы покажем, что опустить это условие нельзя. Далее везде (кроме замечания после примера 3 ): $X=[0,1] ; \Sigma_{X}-$ борелевская (или лебеговская, безразлично) $\sigma$-алгебра; $\mu$-мера Лебега; $Y=X^{[0,1]}-$ множество всех функций из $[0,1]$ в $[0,1] ; \Sigma_{Y}=\Sigma_{X}^{[0,1]}-\sigma$-алгебра, порожденная цилиндрическими подмножествами $Y ; \rho=\mu^{[0,1]}-$ мера в $Y$, являющаяся прямым произведением мер $\mu$ (так что $\left(Y, \Sigma_{Y}, \rho\right)=\left(X, \Sigma_{X}, \mu\right)^{[0,1]} ;$ подробнее о несчетных произведениях вероятностных пространств см., например, [2]); $L_{2}=L_{2}\left(Y, \Sigma_{Y}, \rho\right)$ - гильбертово пространство квадратично интегрируемых относительно $\rho$ действительных функций на $Y$; $e_{x} \in L_{2}$ : $e_{x}(y)=\sqrt{2} \cos \pi y(x)(x \in X)$. Основой приводимых ниже примеров 1-3 является следующее предложение. 
Лемма. Для функиии

$$
\nu: X \times \Sigma_{Y} \rightarrow[-1,1]: \nu(x, A)=\left(e_{x}, 1_{A}\right)=\int_{A} e_{x}(y) \rho(d y)
$$

справедливы включения $\nu \in M(X, Y),|\nu| \in M(X, Y)$.

ДоказАтельство. Так как $\left\{e_{x}: x \in X\right\}$ есть ортонормированная в $L_{2}$ система, то (неравенство Бесселя) для любого $A \in \Sigma_{Y}$ существует лишь счетное множество точек $x \in X$ таких, что $\left(e_{x}, 1_{A}\right) \neq 0$. Отсюда сразу следует включение $\nu \in M(X, Y)$ и равенство

$$
\nu \times \mu=0 .
$$

Пусть $A \in \Sigma_{Y}$. Тогда [2, III.3] существует множество $X_{0}=X_{0}(A)$ такое, что: 1$)$ множество $X \backslash X_{0}$ счетно; 2) для любых $y, z \in Y$ справедлива импликация

$$
(y \in A) \wedge\left(y(x)=z(x) \forall x \in X \backslash X_{0}\right) \Longrightarrow z \in A .
$$

Для точек $x_{1}, x_{2} \in X_{0}$ рассмотрим отображение $T: Y \rightarrow Y: T y(x)=y(x)$, если $x \neq x_{1}$, $x \neq x_{2} ; T y\left(x_{1}\right)=y\left(x_{2}\right) ; T y\left(x_{2}\right)=y\left(x_{1}\right)$. Ясно, что отображение $T$ измеримо. Представляя $\left(Y, \Sigma_{Y}, \rho\right)$ в виде $\left(X, \Sigma_{X}, \mu\right)^{2} X^{[0,1]} \backslash\left\{x_{1}\right\} \cup\left\{x_{2}\right\}$ и пользуясь инвариантностью меры $\mu^{2}$ при ортогональных преобразованиях квадрата (точнее: при отражениях относительно биссектрисы), получаем, что отображение $T$ сохраняет меру $\rho: \rho\left(T^{-1} B\right)=\rho(B)$ $\forall B \in \Sigma_{Y}$, так что для индуцированной отображением $T$ меры $T \rho$ справедливо равенство $T \rho=\rho$. Отсюда, из формулы замены переменных в интеграле, импликации (7) и включения $x_{1}, x_{2} \in X_{0}$ получаем

$$
\begin{aligned}
|\nu|\left(x_{1}, A\right) & =\int_{A}\left|e_{x_{1}}(y)\right| \rho(d y)=\int_{A}\left|e_{x_{1}}(y)\right| T \rho(d y) \\
& =\int_{T^{-1} A}\left|e_{x_{1}}(T y)\right| \rho(d y)=\int_{A}\left|e_{x_{2}}(y)\right| \rho d y=|\nu|\left(x_{2} A\right) .
\end{aligned}
$$

Таким образом, функция $x \mapsto|\nu|(x, A)$ постоянна на множестве $X_{0}$ и, следовательно, измерима. Лемма доказана.

ПримеР 1. Возьмем переходную меру $\nu$, определенную равенством (5). Для любого $x \in X$ имеем

$$
|\nu|(x, Y)=\int_{Y}\left|e_{x}(y)\right| \rho(d y)=\sqrt{2} \int_{0}^{1}|\cos \pi t| \mu(d t)=\frac{2 \sqrt{2}}{\pi} .
$$

Если теперь $g: X \rightarrow[-\infty, \infty]$ есть функция, равная $\infty$ на множестве положительной $\mu$-меры, то полагая $f: X \times Y \rightarrow[-\infty, \infty]: f(x, y)=g(x)$, из (6) получаем $\nu \times \mu$-интегрируемость $f$. Но в силу (8) заключение о $\nu(x, \cdot)$-интегрируемости функции $y \mapsto f(x, y)$ $\mu$-почти при всех $x$ неверно, т.е. нарушается первое утверждение теоремы Фубини.

ЗАмечАниЕ. Вьшеприведенная формулировка теоремы Фубини может быть изменена так, что требование $\nu \times \mu$-интегрируемости $f$ ослаблено требованием ее $\overline{\nu \times \mu}$-интегрируемости (пространство $\left(X \times Y, \overline{\Sigma_{X} \times \Sigma_{Y}}, \overline{\nu \times \mu}\right)$ есть пополнение пространства $\left(X \times Y, \Sigma_{X} \times \Sigma_{Y}, \nu \times \mu\right)$ по мере $|\nu \times \mu|$, см. $[1, \S 5$, п. 4]). $\mathrm{K}$ такой измененной теореме возможно построение более содержательных контрпримеров. Именно, беря $\nu$ ту же, что и вьше, и полагая последовательно $f(x, y)=1 / y(x), f(x, y)=(1 / x) \cos \pi y(x)$, $f(x, y)=\cos \pi y(x)$ получим соответственно нарушение первого, второго и третьего утверждений теоремы. 
ПримеР 2. Пусть $B \subset X$ есть какое-то неизмеримоемножество. Положим $\nu: X \times \Sigma_{Y}$ $\rightarrow[-1,1]: \nu(x, A)=1_{B}\left(e_{x}, 1_{A}\right)$; так же, как и при доказательстве леммы получаем $\nu \in M(X, Y)$. С другой стороны (см. равенство (8)), $\{x \in X:|\nu|(x, Y)>0\}=B$, так что функция $x \mapsto|\nu|(x, Y)$ неизмерима, т.е. не выполняется (3).

ПРИмеР 3. Опять возьмем $\nu$, определенную равенство (5); в силу второго утверждения леммы мера $|\nu| \times \mu$ корректно определена. Теперь (8) дает равенство $|\nu| \times \mu(X \times Y)=$ $2 \sqrt{2} / \pi$, a $(6)-$ равенство $|\nu \times \mu|=0$. Таким образом, не вьполняется (4).

ЗАмечаниЕ. Пусть $\left(X, \Sigma_{X}\right),\left(Y, \Sigma_{Y}\right)$ - измеримые пространства, $\nu \in M(X, Y)$, $x \in X, A^{+}(x)$ - положительная часть разложения Хана для меры $\nu(x, \cdot) \in M(Y)$. Множество $A^{+}(x)$ определено с точностью до $|\nu|(x, \cdot)$-меры нуль. Пример 3 показывает, что, вообще говоря, нельзя для каждого $x \in X$ выбрать $A^{+}(x)$ так, чтобы выполнялось включение $\bigcup_{x}\left\{x, A^{+}(x)\right\} \in \Sigma_{X} \times \Sigma_{Y}$.

Перед следующим примером заметим, что если $\mu \in M^{+}(x), \nu \in M^{+}(X, Y)$, то произведение $\nu \times \mu$ всегда корректно определено равенством (2) (интегралу "разрешено" принимать значение $+\infty)$; при этом $\nu \times \mu \in M^{+}(X \times Y)$. Напомним еще, что мера $\rho \in M^{+}(Z)(Z$ - измеримое пространство) называется $\sigma$-ограниченной, если существует последовательность измеримых множеств $Z_{n} \subset Z$ такая, что $Z=\cup Z_{n}$ и для всех $n$ $\rho\left(Z_{n}\right)<\infty$. Есть соответствующее определение и для векторных мер. В [1] (для векторного случая) требовалась $\sigma$-ограниченность мер $\nu(x, \cdot)(\forall x \in X), \nu \times \mu$. Естественен вопрос: вытекает ли $\sigma$-ограниченность $\nu \times \mu$ из $\sigma$-ограниченности $\nu(x, \cdot), \mu ?$

Пример 4. Для $y \in Y$ обозначим через $\delta_{y} \in M^{+}(Y)$ меру (Дирака) единичной массы, сосредоточенную в точке $y$. Для $x \in X$ через $1_{x} \in Y$ обозначим индикаторную функцию одноточечного множества $\{x\}$. Пусть $\left\{r_{n}\right\}$ есть множество всех рациональных точек отрезка [0, 1]. Положим $\nu: X \times \Sigma_{Y} \rightarrow[0, \infty]: \nu(x, A)=\sum_{n=1}^{\infty} \delta_{r_{n} 1_{x}}(A)$. Пусть $A \in \Sigma_{Y}, X_{0}=X_{0}(A)$ - то же, что и в доказательстве леммы. Если $0 \in A$, то для всех $x \in X_{0}, n=1,2, \ldots$, выполняется включение $r_{n} 1_{x} \in A$, откуда $\nu(x, A)=\infty$. Если $0 \notin A$, то, аналогично, $\nu(x, A)=0$ для всех $x \in X_{0}$. Таким образом, в обоих случаях функция $x \mapsto \nu(x, A)$ измерима, т.е. $\nu \in M^{+}(X, Y)$. Полагая для $x \in X$ $Y_{n}=\left\{y \in Y: y(x)=r_{n}\right\}, Y_{0}=Y \backslash \bigcup_{n=1}^{\infty} Y_{n}$ видим, что $\nu\left(x, Y_{0}\right)=0, \nu\left(x, Y_{n}\right)=1$, так что мера $\nu(x, \cdot) \sigma$-ограничена.

Пусть $B \in \Sigma_{X} \times \Sigma_{Y}$. Снова пользуясь результатом [2, III.3] (теперь применительно к пространству $\left.\left(X \times X^{[0,1]}, \Sigma_{X} \times \Sigma_{X}^{[0,1]}\right)\right)$, возьмем множество $X_{0} \subset[0,1]$ со счетньм дополнением такое, что сечение $S_{x}(B)$ не зависит от $x$ при $x \in X_{0}$. Обозначая это общее сечение через $A \in \Sigma_{Y}$, получим равенство $\nu \times \mu(B)=\nu \times \mu(X \times A)=\int_{0}^{1} \nu(x, A) \mu(d x)$. Но вьше было найдено, что либо $\nu(x, A)=\infty \mu$-почти при всех $x$, либо $\nu(x, A)=0$ $\mu$-почти при всех $x$. Таким образом, либо $\nu \times \mu(B)=\infty$, либо $\nu \times \mu(B)=0$, так что мера $\nu \times \mu$ не является $\sigma$-ограниченной.

\section{СПИСОК ЦИТИРОВАННОЙ ЛИТЕРАТУРЫ}

[1] Угланов А. В. Теорема Фубини для векторных мер // Матем. сб. 1990. Т. 181.№3. C. 423-432.

[2] Неве ЖК. Математические основы теории вероятностей. М.: Мир, 1969. 